The Egyptian Journal of Hospital Medicine (July 2019) Vol. 76 (5), Page 4202-4212

\title{
Evaluation of Chandelier Assisted Pars Plana Vitrectomy for Management of Epiretinal Membranes
}

\author{
Abdelhafez, Y., ${ }^{(1)}$ Mohamed, I. A., ${ }^{(2)}$ Ghali, A. ${ }^{(1)}$ and Omran, M. ${ }^{(1)}$ \\ (1) Department of Ophthalmology, Faculty of Medicine Al-Azher University, Damietta \\ (2) Department of Ophthalmology, Faculty of Medicine Al-Azher University Cairo \\ Corresponding author: Mohamed Y. Omran, E.mail:my.omran22@Gmail.com, Phone: 00201010941536
}

\begin{abstract}
Background: ERM is a vision affecting disorder, frequently developed secondary to advanced PDR. DR remains one of the leading causes of legal blindness in the working- age population.

Aim of the work: evaluation the safety, efficacy and feasibility of the chandelier assisted PPV for management of fibro-vascular membranes.

Patients and methods: 43 eyes in 36 patients presented with ERM of different etiologies. After full ophthalmic examination, all patients underwent chandelier assisted PPV with silicone oil or gas tamponade without or with phacoemulsification and posterior chamber IOL implantation.

Results: Combined phacoemulsification and PPV was done in 12 cases (27.9\%), 2 cases were Pseudophakic while lens sparing PPV was done in 29 cases (67.4\%). Complete ERM removal was achieved in 38 cases (88.4\%) and incompletely removed in five cases $(11.6 \%)$. Residual tractional RD was observed in two cases $(4.7 \%)$. Iatrogenic breaks were occurred in 7 cases (16.3\%). Endo-laser photocoagulation was done in 33 cases (76.7\%). Silicone oil tamponade was used in 33 cases $(76.7 \%)$, gas in 8 cases $(18.6 \%)$ while air tamponade was used in 2 cases $(4.7 \%)$. There was improvement of BCVA in 31 cases $(72.1 \%)$, while 10 cases $(23.3 \%)$ remained the same and worsened in 2 eyes $(4.7 \%)$ due to recurrent proliferation, consecutive optic disc atrophy and development of neo-vascular glaucoma.

Conclusion: Chandelier assisted bimanual PPV is an effective, safe and an attainable technique for management of ERM, learning curve with this technique and coast benefit ratio may be considered.

Keywords: Chandelier, Bimanual vitrectomy, Epiretinal membrane.
\end{abstract}

\section{INTRODUCTION}

Epiretinal membrane (ERM) is a common disorder of the vitreoretinal interface defined as fine, semi-translucent, fibro-vascular proliferation on the inner retinal surface resulting in visual impairment of variable degrees ${ }^{(1)}$. ERM can be classified as primary "idiopathic" or secondary to another etiology either systemic disease or ocular pathology such as posterior uveitis ${ }^{(2)}$, cytomegalovirus retinitis ${ }^{(3)}$, retinal vein occlusion, blunt force trauma, retinal detachment and repair, argon laser photocoagulation, cataract surgery ${ }^{(4,5)}$ and diabetic retinopathy (DR) which is considered the commonest cause of ERM worldwide ${ }^{(6)}$.

The exact pathogenesis of ERM is still unknown but presence of inflammatory cells with involvement of cellular proliferation, migration, and adhesion suggests that secondary ERM formation may be as an abnormal wound healing response ${ }^{(7,8)}$.

The patient with ERM has variable clinical presentation; may be completely asymptomatic, diagnosed on routine examination, or present with impairment of vision in the form of metamorphopsia, micropsia or macropsia, photopsia, decreased visual acuity (VA), up to loss of central vision ${ }^{(5,8)}$. Usually cases with Cellophane maculopathy are asymptomatic while more severe forms of ERM cause significant visual symptoms impacting the quality of life up to visual loss ${ }^{(9)}$.

The clinical significance of ERM is the tendency of membrane to thicken and contract leading to retinal distortion and the appearance of superficial retinal folds or traction lines, becoming opaque and gray. All these will further induce macular edema, vitreous hemorrhage $(\mathrm{VH})$ and even tractional retinal detachment (TRD), these complications may severely damage patient's visual function ${ }^{(6)}$.

Clinical examination of pre-retinal macular fibrosis reveals a semi-translucent membrane obscuring the underlying retinal features and may be associated with superficial or full-thickness retinal folds or traction lines and vascular tortuosity or dilation. Severe forms of ERMs can involve retinal hemorrhages, exudates, vascular abnormalities, edema, macular pseudo-holes, and macular holes, resulting in vision related disabilities (4).

Pars plana vitrectomy with ERM peeling is the standard treatment of the ERM which restore structure with frequently residual visual impairment $(10,11)$. Surgical outcome is influenced by a number of factors including the patient age, location and extent of the TRD, and the duration of macular heterotopia. Older 
age, anterior segment neovascularization, longstanding macular distortion have been associated with poorer visual outcomes ${ }^{(12)}$. Meticulous sectioning and removal of fibro-vascular membranes is essential step which is considered the most critical and challenging event that may be associated with severe complications especially in treating severe PDR but fortunately was improved after great advancement in vitreoretinal surgery. Membrane peeling with several scissors or special instruments, maneuvered with one hand or bimanually, was used ${ }^{(13)}$.

Challenging cases of extensive tight fibrovascular ERMs during vitreoretinal operations can be better operated using bimanual approach than with conventional single-handed methods, that is because the bimanual method decrease of unwanted pull and pushout forces produced by instruments during ERM peeling ${ }^{(14)}$.

Variable methods and instruments were described to be used in bimanual membrane dissection in previous studies ${ }^{(15)}$, for example, the selfillumination system, the multiport illumination system, and the optical fiber-free intravitreal surgery system (OFISS). However, the self-illuminated instruments are expensive, require larger sclerotomy openings, have limited choices, express greater retinal photo-toxicity with restricted area of illumination as the light goes very close to the retinal tissue ${ }^{(16)}$.

Multiport illumination system has also some limitations as larger sclerotomies (20-gauge cannulas), inability to use many-angled instruments, a longer initial setup time, and additional time for scleral sutures (15). Optical fiber-free intravitreal surgery system (OFISS) is another method for bimanual PPV which provides an excellent visualization of tissues and give the surgeon the chance to manipulate freely with both hands, but OFISS needs special equipment, and it provides poor fundus visualization in phakic and pseudophakic eyes during fluid-gas exchange ${ }^{(17)}$.

Four port PPV using chandelier illumination system as the source of endo-illumination for bimanual vitrectomy is an essential part of modern minimal incision vitreoretinal surgery as it can provide excellent fundus visualization including peripheral vitreous, and provides panoramic wide bright illumination for bimanual surgery (Eckardt, 2003) ${ }^{(14)}$. Furthermore, chandelier system express less retinal photo-toxicity allowing the surgeon to operate with two active hands, more reliability for ERM peeling with the ability to counteract the traction upon the retina with less incidence of intraoperative iatrogenic retinal breaks, more control of bleeding during ERM dissection and giving the surgeon chance for self-indentation ${ }^{(14,18)}$.
Chandelier system can also give the surgeon an extra-advantage during combined vitrectomy and phacoemulsification operation in cases with vitreous hemorrhage with dim red reflex as it is effective in providing retro-illumination during cataract surgery. It is of great help in many phacoemulsification steps as capsulorhexis and irrigation aspiration of cortical matter (19).

The aim of the current study was to evaluate safety, efficacy and feasibility of the chandelier assisted pars plana vitrectomy for management of fibro-vascular membranes in different vitreoretinal pathology as regards the anatomical success and functional outcome.

\section{PATIENTS AND METHODS}

This study included a total of 43 eyes in 36 patients (7 patients bilateral) presented with epiretinal membrane (ERM) of different etiologies attending at Department of Ophthalmology, Faculty of Medicine, Al-Azhar University Hospital, Damietta, Egypt. This study was conducted between Mars 2016 and December 2018.

\section{Ethical approval:}

Approval of the ethical committee and a written informed consent from all the subjects were obtained.

Inclusion criteria: Patients indicated for PPV with presence of ERM either idiopathic or secondary to proliferative diabetic retinopathy (PDR) with or without tractional retinal detachment (TRD), proliferative vitroretinopathy (PVR), retinal vein occlusion or uveitis.

Exclusion criteria: Presence of sub-retinal pathology indicating sub-retinal surgery, ERM in silicone filled eyes, presence of another factor denoting and/or contributing postoperative poor visual prognosis as neovascular glaucoma, optic disc atrophy, supra-choroidal hemorrhage, patient refused to be included in the study and lack of regular follow up.

Preoperative preparation: Full history was taken. Ophthalmological clinical examination including: Measurement of best corrected visual acuity (BCVA). Measurement of intraocular pressure (IOP). Slit-lamp examination of the anterior segment and slit lamp biomicroscopy. Fundus photography if there was clear media. B-scan ultrasonography in opaque media. biometry for all phakic cases.

Preoperative laboratory investigation: Complete blood count, fasting blood glucose, fasting and 2 hour postprandial blood glucose, coagulation profile, liver 
ejhm.journals.ekb.eg

and kidney function tests were done to exclude ineligible cases.

Operations were done by the same surgeon (Ghali, A.) under general or local anesthesia according the patient age, and fitness. Preoperative anti-VEGF (Bevacizumab with dose of $2.5 \mathrm{mg}(0.1 \mathrm{ml})$ [Avastin; Genentech, Inc., San Francisco, CA]) was injected under complete a septic condition in the operating theater in selected cases.

Surgical steps: Chandelier assisted PPV (4-port bimanual PPV) using trocar system (23 G system for infusion and instrumentation (Fig. 1) at infero-temporal, supero-temporal and nasal while the chandelier was inserted at 6 O'clock $(25 \mathrm{G})$, the sclerotomies was done at 3.5- $4 \mathrm{~mm}$ from the corneal limbus (Fig. 1).

Fundus visualization was achieved using [OCULUS BIOM®5 or Ziess RESIGHT ®700] held on surgical microscope.

Phacoemulsification with posterior chamber IOL (PC-IOL) was done in cases with planned combined phacoemulsification-PPV, in cases with inappropriate red reflex (R.R), Trypan-blue assisted capsulorhexis with the use of chandelier retroillumination as alternative to the patient R.R to complete phacoemulsification steps. In some cases, gaining of the patient's R.R was achieved by core vitrectomy before starting of phacoemulsification steps.

Starting with the ordinary light, core vitrectomy assisted by Tri-Amcinol Acetenoid (TAA) was done using parameters; cutting 3500-5000 cuts/minute, vacuum $250-450 \mathrm{mmHg}$, if induced posterior vitreous detachment (PVD) could not be achieved, segmentation of the posterior hyaloid membrane was done to remove the adhesions between the retina and the vitreous base before tackling of the ERM, anterior hyaloid face is then removed using the red reflex with microscope without using the BIOM system in order to gain clear media before ERM peeling.

Chandelier was used instead of the ordinary light as a source of illumination with the start of ERM peeling, ERM was then operated bimanually using curved scissors and either of end-gripping forceps or cutter in suction mode, the assistant maintained direction of the chandelier light to gain the maximum illumination focused at the scene of the ERM peeling.

More than one technique were used in removal of the fibro-vascular membranes as membrane dissections, segmentation and en bloc excision (Fig. 1). Dealing with residual un-dissectible fibro-vascular tissue was done by segmentation of the ERM between the traction points followed by trimming using the vitreous cutter in shaving mode (cutting 5000 cuts/minute, vacuum $150 \mathrm{mmHg}$ ) to avoid more traction on the retina especially in dangerous areas as around the macula.

Bleeding was controlled by simultaneous use of intraocular diathermy for cauterization of the bleeding point and the cutter in suction mode to decrease the cloudiness occurred by hemorrhage on the scene of maneuver. Dealing with accumulated blood was done using the soft tip back flush simultaneously with active suction by the cutter probe with increasing the IOP.

Fluid-air exchange and drainage of sub-retinal fluid through iatrogenic retinal break or retinotomy (Fig. 1). Endo-laser application was done according to the need. The number of laser shots is determined according to retinal status (Fig. 1). The choice of the proper tamponade between silicone oil, sulfur hexafluoride $20 \%$ (SF6, expansible gas) or air was selected according to the retinal situation at the end of the surgery.

Postoperative follow up was done for all patients at 1 day postoperative, 1 week, 1 month and then monthly for 6 months after the surgery. At each follow-up visit full ophthalmological examination was done including: BCVA, IOP, anterior segment and fundus examination.

\section{Statistical analysis}

Data was analyzed using IBM SPSS Advanced Statistics version 20.0 (SPSS Inc., Chicago, IL). Numerical data were expressed as mean and standard deviation. Qualitative data were expressed as frequency and percentage.

\section{RESULTS}

Forty three eyes of thirty sex patients were included in the study, summery of demographic data are included in table 1. All cases had ERM of different etiologies (Fig. 2) and had been indicated for PPV, but the main indication for PPV was different (Table 2). Combined phacoemulsification and PPV was done in 12 cases (27.29\%); phacoemulsification depending on the patient's own R.R in 10 cases, 2 cases had dense vitreous hemorrhage in which phacoemulsification was assisted with Trypan-blue staining of the anterior capsule as well as use of the chandelier retroillumination. Two cases (4.7\%) were pseudophakic and 29 cases $(67.4 \%)$ underwent lens sparing PPV(LSPPV) due to clear lens or faint cataract not disturbing the visualization. 

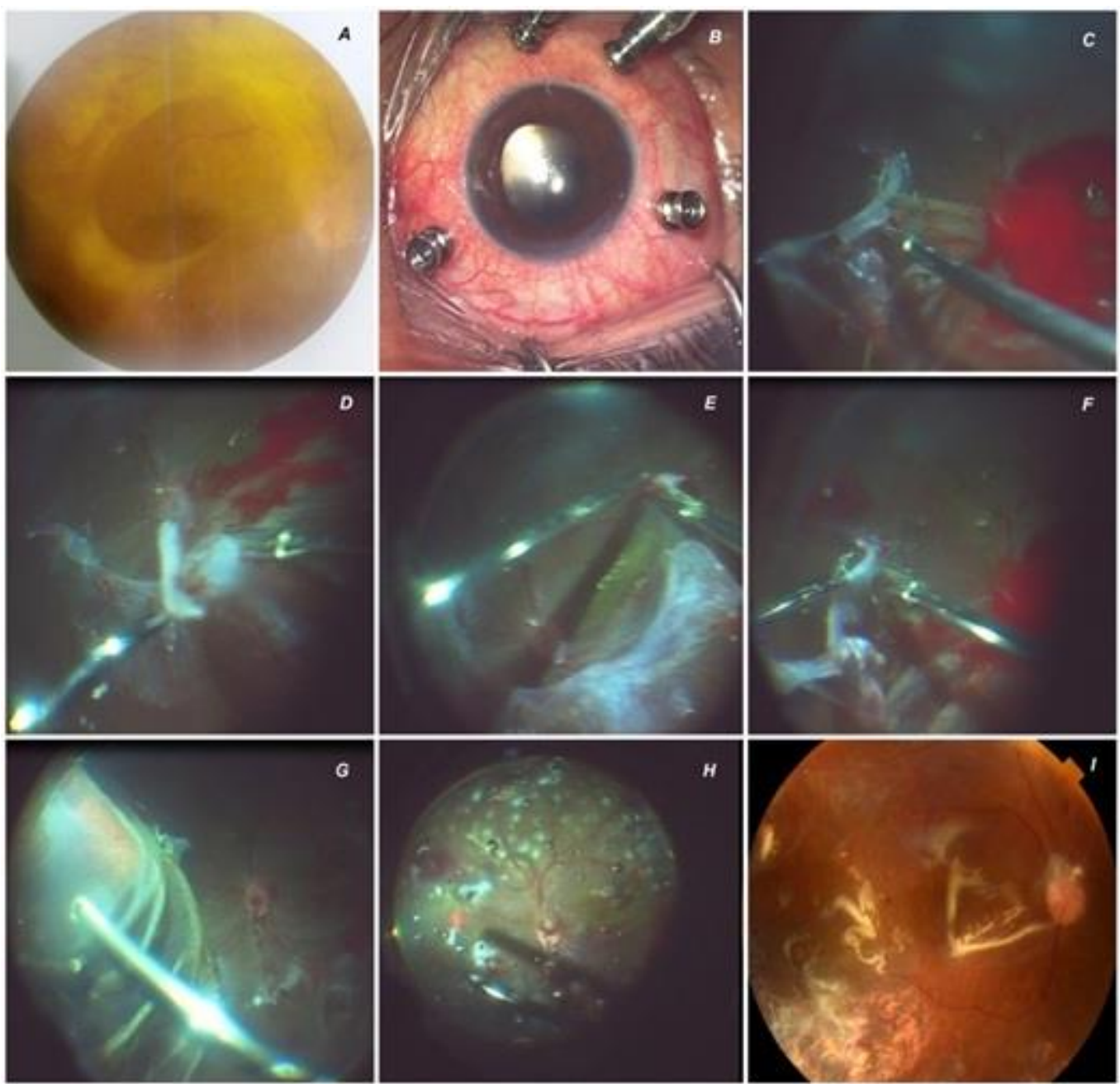

Figure (1): The plate shows preoperative (A), intraoperative steps (B-H) and postoperative fundus photos (I) for 49 years old lady with advanced diabetic eye disease, ERM and TRD.

Table (1): Demographic data of patient included in the study

\begin{tabular}{|c|c|c|c|}
\hline & Characteristics & Number & Percentage \\
\hline Age & $\begin{array}{l}\text { Min.-max. } \\
\text { Mean }\end{array}$ & $\begin{array}{l}19-75 \text { years } \\
53.9 \text { years }\end{array}$ & \\
\hline \multirow[t]{3}{*}{ Sex } & $\begin{array}{l}\text { Male } \% \text { - Eyes } \% \\
\text { Female } \% \text { - Eyes } \%\end{array}$ & $\begin{array}{l}14(38.9 \%) \\
22(61.1 \%)\end{array}$ & $\begin{array}{l}15(34.9 \%) \\
28(65.1 \%)\end{array}$ \\
\hline & Type I Diabetes Mellitus & \multicolumn{2}{|l|}{$8(18.6 \%)$} \\
\hline & Type II Diabetes Mellitus & \multicolumn{2}{|l|}{$33(67.7 \%)$} \\
\hline \multirow[t]{6}{*}{ Duration Of D.M. } & $\begin{array}{l}\text { Min.- Max. } \\
\text { Mean }\end{array}$ & $\begin{array}{l}\text { 12-30Years } \\
\text { 19.2 Years }\end{array}$ & \\
\hline & Hyperlipidemia & 9 & $(25 \%)$ \\
\hline & Hypertension & 12 & $(33.3 \%)$ \\
\hline & Cardiac & 10 & $(27.8 \%)$ \\
\hline & Aspirin therapy & 7 & $(19.4)$ \\
\hline & Renal impairment & 3 & $(8.3 \%)$ \\
\hline Laterality & $\begin{array}{l}\text { Right eye (OD) } \\
\text { Left eye (OS) } \\
\text { Bilateral (OU) }\end{array}$ & $\begin{array}{l}20 \text { Eyes } \\
23 \text { Eyes } \\
7 \text { patients }\end{array}$ & $\begin{array}{l}46.5 \% \\
53.5 \% \\
19.4 \% \\
\end{array}$ \\
\hline
\end{tabular}




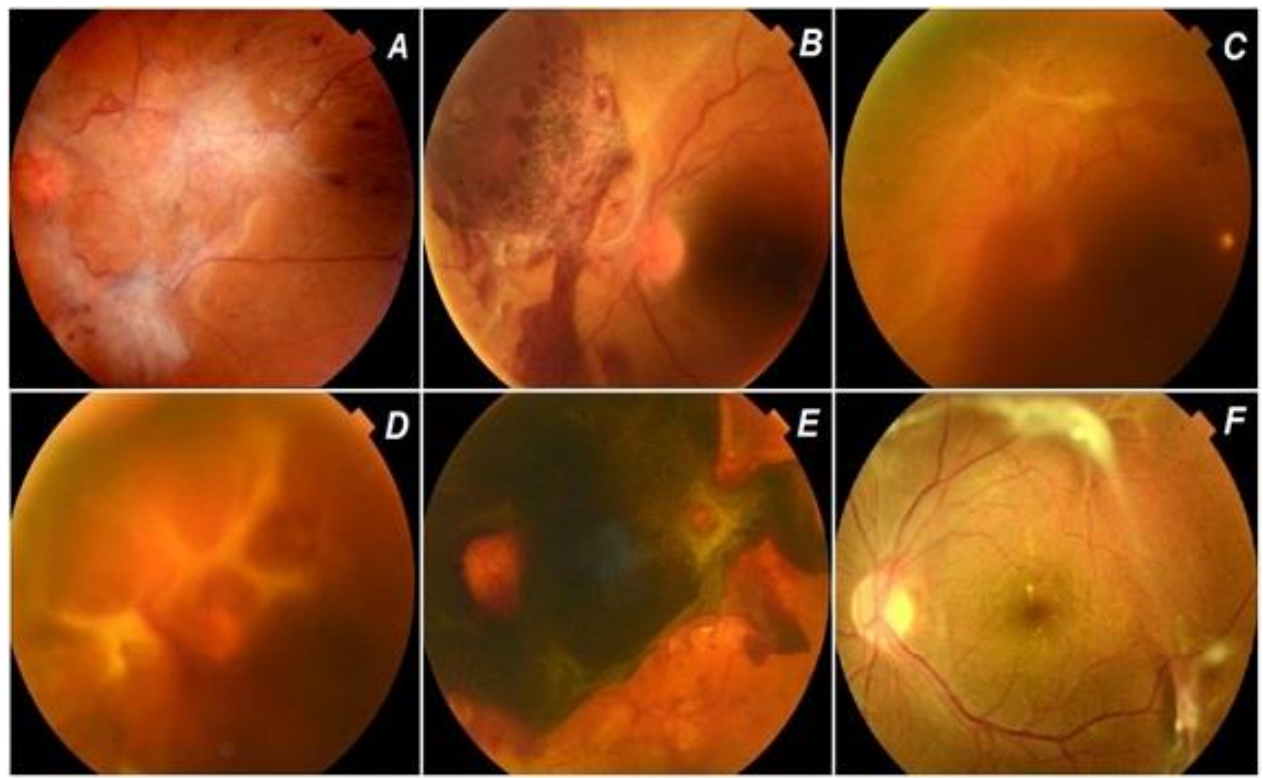

Figure (2): Preoperative fundus photos for some cases involved in the study.

Table (2): Preoperative data as regards indication of PPV, lens status, procedure.

\begin{tabular}{|c|c|c|c|c|}
\hline \multicolumn{3}{|c|}{ Characteristics } & No. & $\%$ \\
\hline \multicolumn{5}{|c|}{ 1- $\quad$ Main Indication of vitrectomy } \\
\hline \multirow[t]{5}{*}{ 1.1- } & \multicolumn{2}{|c|}{ Tractional retinal detachment } & 26 & $60.5 \%$ \\
\hline & 1.1 .1 & TRD involving macula & 12 & $27.9 \%$ \\
\hline & 1.1 .2 & TRD threatening the macula & 11 & $25.6 \%$ \\
\hline & 1.1 .3 & TRD not involving the macula & 2 & $4.7 \%$ \\
\hline & 1.1.4 & $\begin{array}{l}\text { Combined } \\
\text { rhegmatogenous RD }\end{array}$ & 1 & $2.3 \%$ \\
\hline \multirow[t]{3}{*}{ 1.2- } & \multicolumn{2}{|c|}{ ERM with persistent VH } & 14 & $32.6 \%$ \\
\hline & 1.2 .1 & On top of PDR & 13 & $30.2 \%$ \\
\hline & 1.2 .2 & On top of Vein Occlusion & 1 & $2.3 \%$ \\
\hline 1.3- & \multicolumn{2}{|c|}{ Vitritis associated with ERM } & 1 & $2.3 \%$ \\
\hline 1.4- & \multicolumn{2}{|c|}{ Premacular sub-ERM he } & 1 & $2.3 \%$ \\
\hline $1.5-$ & \multicolumn{2}{|c|}{ ERM causing macular bucker } & 1 & $2.3 \%$ \\
\hline 2- & \multicolumn{4}{|c|}{ Lens status } \\
\hline 2.1 & \multirow{2}{*}{\multicolumn{2}{|c|}{$\begin{array}{l}\text { Phakic } \\
\text { Pseudophakic }\end{array}$}} & 41 & $95.3 \%$ \\
\hline 2.2 & & & 2 & $4.7 \%$ \\
\hline \multicolumn{5}{|c|}{ 3- $\quad$ Procedure } \\
\hline 3.1 & \multicolumn{2}{|c|}{ Lens sparing PPV } & 29 & $(67.4 \%)$ \\
\hline 3.2 & \multicolumn{2}{|c|}{ Pseudophakic PPV } & 2 & $(4.7 \%)$ \\
\hline 3.3 & \multicolumn{2}{|c|}{ Phaco- PPV } & 12 & $(27.9 \%)$ \\
\hline
\end{tabular}

Epiretinal membrane was completely removed (Fig. 3 and 5) in 38 cases $(88.4 \%)$ while incomplete removal were achieved (Fig. 4 and 6) in five cases $(11.6 \%)$; none of them significantly was affecting the macular area. Iatrogenic breaks were inevitable and occurred but well managed in 7 cases $(16.3 \%)$, and used for drainage of SRF drainage after fluid-air exchange (Fig. 1), there was no indication for retinectomy. Residual TRD was observed in two cases $(4.7 \%)$. Endo-laser photocoagulation (Fig. 1 and 4) was done in 33 cases
(76.7\%) with minimum about 512 shots and maximum 1196 shots, while in the remaining 10 cases $(23.3 \%)$ there was no need for intraoperative endo-laser.

The proper tamponade was selected at the end of each case, silicone oil was selected in 33 cases $(76.7 \%)$ while sulfur hexafluoride (SF6) gas was selected in 8 cases $(18.6 \%)$ and air was selected in 2 cases $(4.7 \%)$. Bevacizumab was injected at the end of the surgery in 38 cases $(88.4 \%)$ to decrease the vitreous cavity hemorrhage 
in the early postoperative period and progression of rubeosis.

Early postoperative vitreous cavity hemorrhage (POVCH) was observed in 8 cases (Fig. 3), medical treatment with systemic workup and control of diabetes mellitus, hypertension and coagulopathy was enough in 7 cases while vitreous cavity wash and silicone oil injection (case with SF6 tamponade) was done in 1 case after 2 weeks with no improvement with medical treatment. Early and late postoperative complications are summarized in (Fig. 8 and 9).

By the end of follow up time, there was improvement of BCVA in $31(72.1 \%)$ cases (Tab. 3, Fig.
7), while remained the same BCVA in 10 cases $(23.3 \%)$ and two eyes $(4.7 \%)$ had worsened BCVA.

Table (3): The final visual outcome after 3 months follow up.

\begin{tabular}{|l|l|l|}
\hline $\begin{array}{l}\text { Visual acuity } \\
\text { after 3 months }\end{array}$ & Number & Percentage \\
\hline $\begin{array}{l}\text { Improved by 2 } \\
\text { lines or more }\end{array}$ & 31 cases & $72.1 \%$ \\
\hline $\begin{array}{l}\text { Remain the same } \\
\text { or improved by } \\
\text { one line or more }\end{array}$ & 10 cases & $23.3 \%$ \\
\hline $\begin{array}{l}\text { Worsened by 2 } \\
\text { lines or more }\end{array}$ & 2 cases & $4.7 \%$ \\
\hline
\end{tabular}

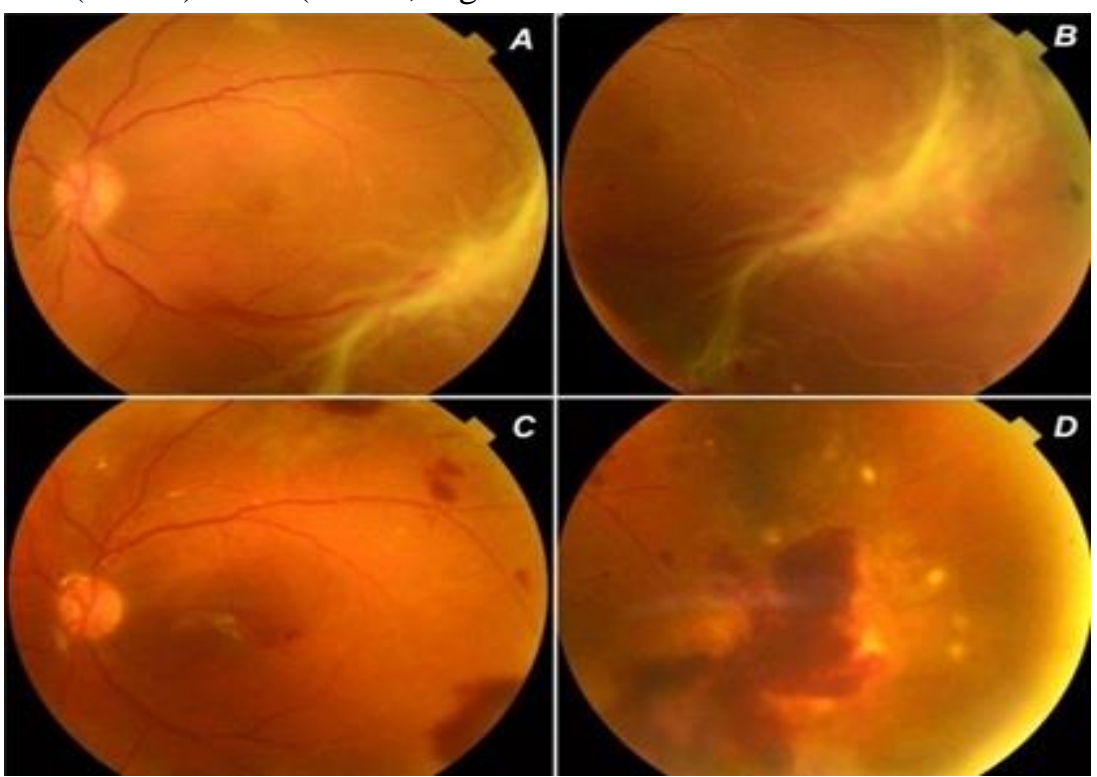

Figure (3): The plate shows fundus photos for 49 years old lady with type II DM for 12 years duration. A and B) Preoperative left colour fundus photos taken at the day of surgery showing advanced DED, ERM with TRD threatening the macula. C and D) 1 weak postoperative fundus photos with retina totally flat under SO with minimal retinal hemorrhage under SO.
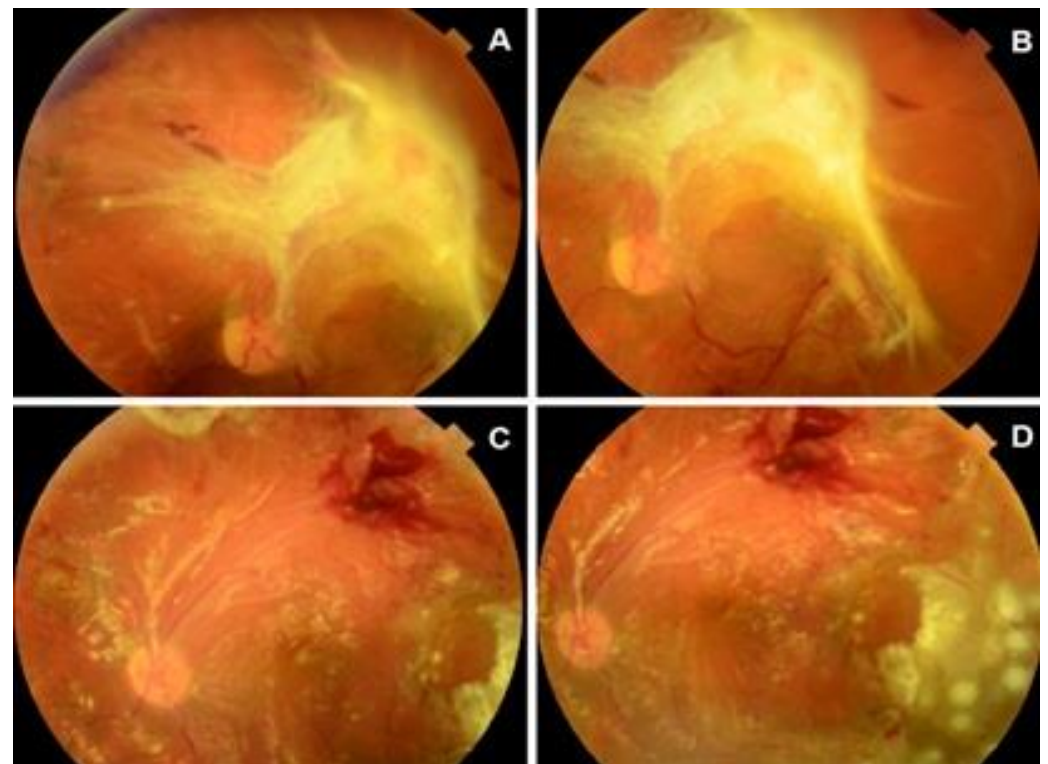

Figure (4): The plate shows left eye pre- and postoperative fundus photos for 23 years old male patient with type-I DM, presenting with extensive ERM, TRD involving the macula (upper photos), the membrane is completely removed, retina completely flat under SO with minimal postoperative hemorrhage. The BCVA was improved from HM (2.3 Log MAR) to 0.1 (1.0 Log MAR). 




Figure (5): The plate shows preoperative (left picture) and postoperative (Right picture) fundus photography for 28 years old lady with type I DM for 21 years old duration, presented with ERM. Bimanual LS-PPV with SF6 was done. The retina was stable postoperative with residual macular pucker.

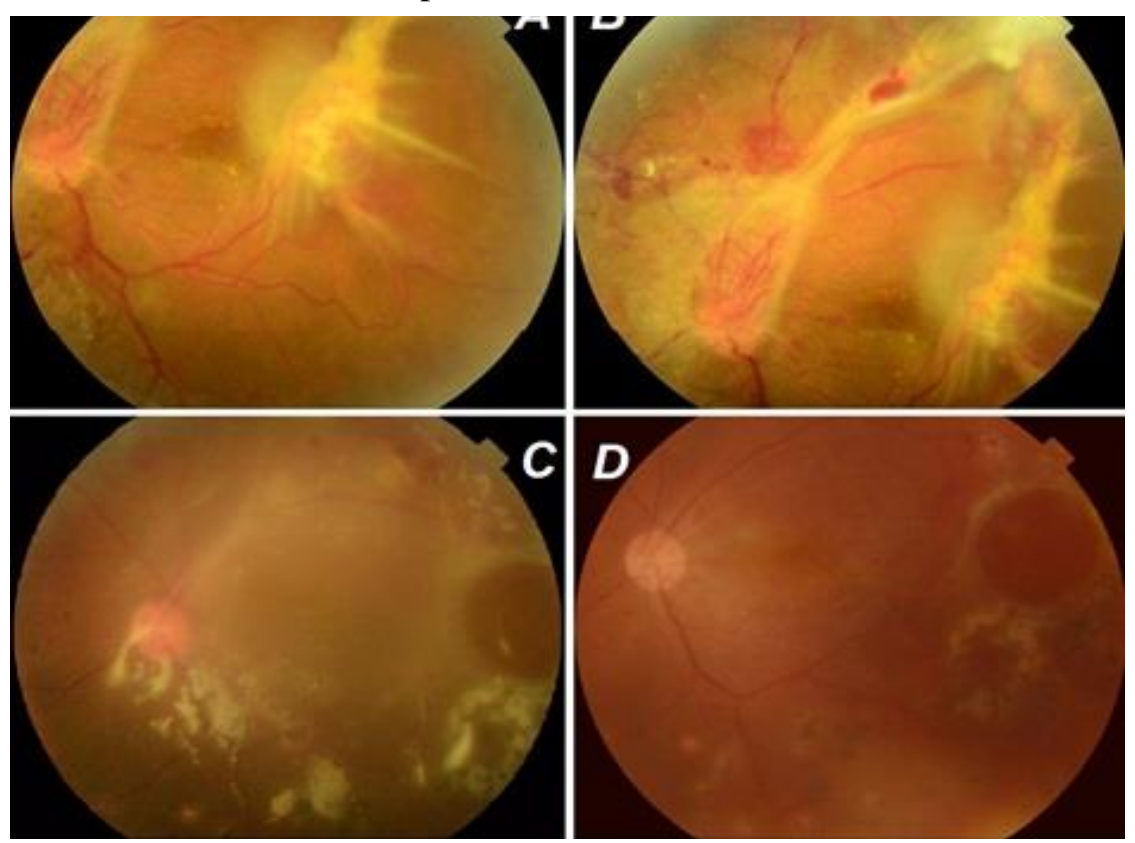

Figure (6): The plate shows 32 years old male patient with IDDM (type I) 22 years duration, presented with bilateral PDR. Right eye had high risk PDR managed by anti-VEGF and PRP. Left eye had advanced DED with ERM, TRD, bimanual PPV and SO was done (A and B). C) Fundus photo 6 months postoperatively with residual ERM, hazy view duo to cataract aggravation. D) Fundus photo taken 1 weak after cataract extraction, IOL and SO removal. The visual acuity improved from $2 / 60$ to 0.2 .

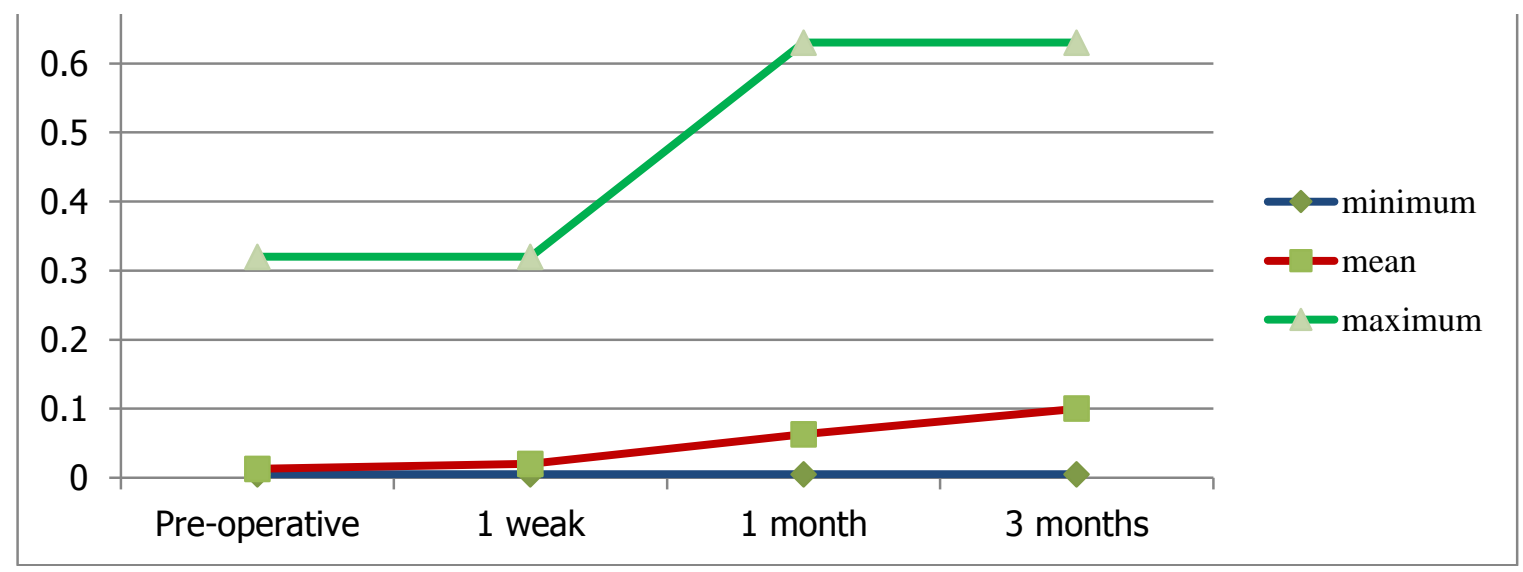


Figure (7) Linear presentation of BCVA (Decimal) as regards the minimum, mean and maximum BCVA in preoperative, 1-week, 1-month and 3-months postoperative follow up.

\section{Early postoperative complications}

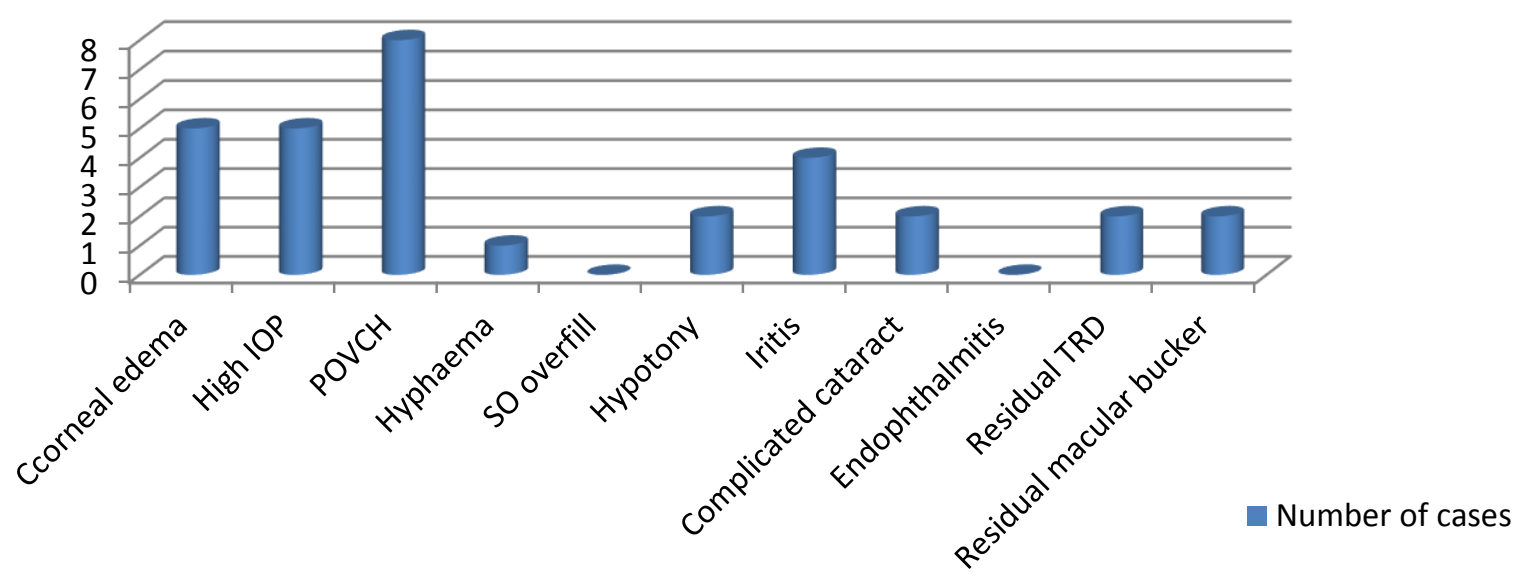

Figure (8): Bar chart illustrating early postoperative complications.

\section{Late postoperative complications}

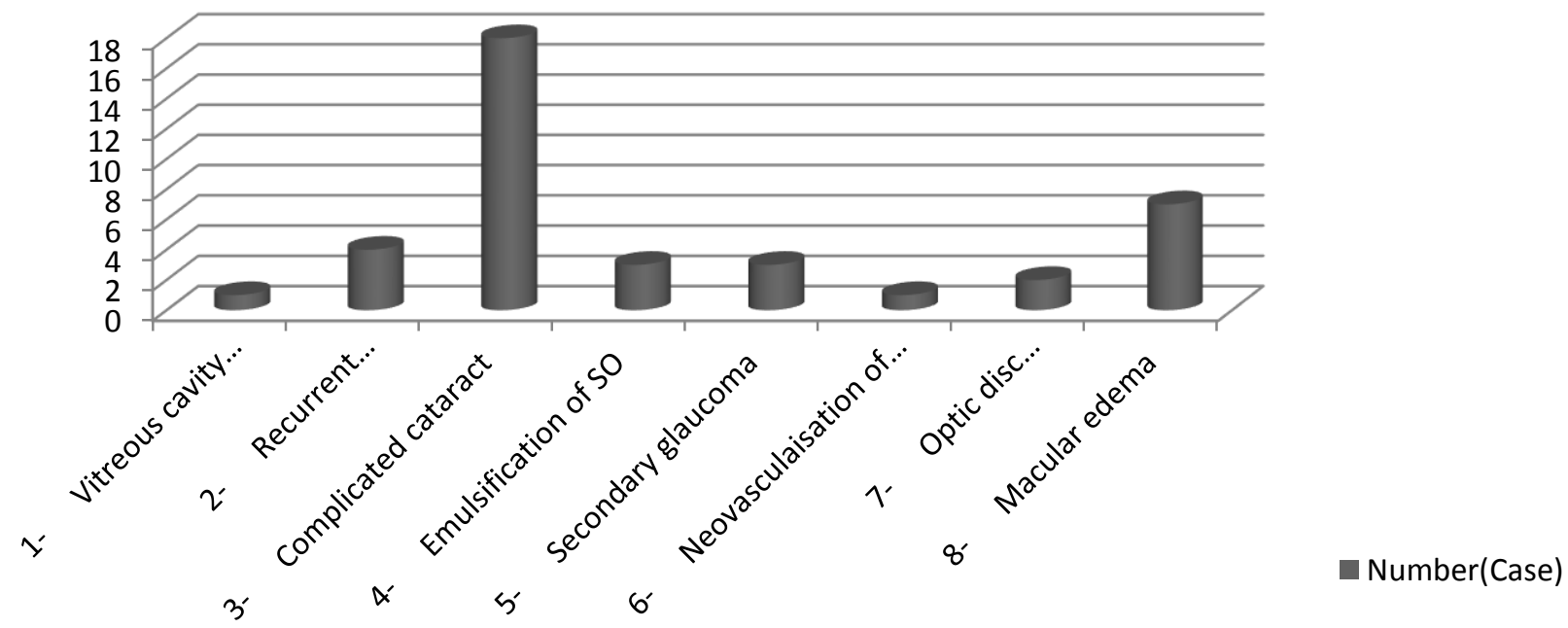

Figure (9): Bar chart illustrating early postoperative complications.

\section{DISCUSSION}

Epiretinal membrane is a disorder of the vitromacular interface that leads to variable degrees of visual impairment by more than one way as disturbance of the visual axis, contraction of epiretinal membrane leading to macular puckering, tractional macular edema, macular hole up to tractional retinal detachment $\left(\mathrm{TRD}^{)}{ }^{(12)}\right.$.

The management options for ERM are currently limited and consist of either observation or surgical intervention, conservative management is the best choice in asymptomatic patients ${ }^{(8,20)}$. Pars plana vitrectomy with ERM peeling is the standard surgical treatment for fibro-vascular membranes as it may relieve tractions and improve vision ${ }^{(21)}$. All surgical innovations for vitrectomy surgeries aimed to find the less invasive and faster method (22). Development of surgical techniques has been led to improvement in anatomic and functional success rates following surgery for severe and challenging cases of ERM ${ }^{(8,23)}$.

Forty three eyes of thirty six patient with ERMs significantly affecting the vision were included in the study for evaluation of chandelier assisted bimanual technique of PPV with ERM peeling, all of them are diagnosed clinically ranged from photopsia, dyschromatopsia and diminution of vision up to visual loss duo to tractional retinal detachment or vitreous hemorrhage associating the ERM. All cases included in 
the study had secondary ERM duo to different etiologies, however diabetic retinopathy is the main epidemiological risk factor in $95 \%$ of cases involved in the study.

Lolah and Shaarawy compared chandelier assisted PPV versus conventional 23 G PPV in a study included sixty patients with diabetic TRD. They concluded that bimanual vitrectomy technique in ERM dissections is more helpful, safe and effective than traditional vitrectomy. Although they suggested that in their results that there is no significant anatomical outcomes, but the bimanual vitrectomy had many befits as decreased surgical time, better control of intraoperative bleeding, less need for intraoperative diathermy and better final visual outcomes after six months of postoperative follow up ${ }^{(18)}$.

The current study evaluated the technique of bimanual PPV assisted by chandelier endo-illumination in cases of ERM; as most of these cases are really challenging because of tight adherence of ERM to retinal surface with high incidence of iatrogenic retinal break. Hence there is no single ideal maneuver for peeling of ERM. The step of ERM sectioning is a highly meticulous necessities good visualization of the scene of peeling. Moreover, ERMs especially in cases of diabetic retinopathy are highly vascularized with high incidence of intraoperative bleeding leading to media haziness and poor visualization during the surgery.

A comparative study between bimanual PPV using (OFFISS) and traditional vitrectomy in eyes with severe PDR was conducted, and it reported full attachment of the retina for all eyes using the bimanual approach 6 months postoperatively ${ }^{(24)}$. The results of the study concluded that the BCVA for 19 of the 22 eyes in the OFFISS group had been improved by two lines or more compared with the control group (17 of the 22 eyes).

In our study, out of forty three cases included in the study, it is statistically evident that there was improvement of BCVA by 2 lines or more in thirty one cases $(72.1 \%)$ while there was no improvement of BCVA (Table 3 ) in ten cases $(23.3 \%)$ and there was worsening of the BCVA in 2 cases, which was explained by ischemic retinopathy and consecutive optic disc atrophy in the two cases but these actually are complication of initial pathogenesis, character and extent of ERM (Fig. 9) and vitrectomy in general not specific for bimanual method for ERM removal.

Occurrence of intraoperative bleeding during pars plana vitrectomy when dissecting epiretinal membrane will seriously affect visualization of the surgical field. Moreover, repeated bleeding can prolong the operation time, increase the frequency of instrument exchange, and greatly increase the occurring rate of complications ${ }^{(25)}$.

Cui et al. on their randomized comparative study on efficacy and safety of intravitreal conbercept, ranibizumab, and triamcinolone on 23-gauge vitrectomy for patients with PDR, approved that the preoperative intravitreal injections of conbercept and ranibizumab had a great effect in improvement of visual acuity, operation time, incidence of intraoperative iatrogenic retinal breaks, endo-diathermy rate, frequency of silicone oil tamponade, vitreous clearing time, and the incidence of intraoperative bleeding. Preoperative injection of anti-VEGF in PPV surgery decreased the vascularization of ERM so the fibrous proliferative membranes were easily separated from the retina with a few individual of bleeding ${ }^{(26)}$.

In our study, preparation with anti-VEGF was done in 26 patients with PDR (60.4\%) at 1-7 days before surgery according the vascularity of ERM, extent and character of ERM as well as presence or absence of TRD.

Iatrogenic breaks in our study occurred in eight cases $(18.6 \%)$ this is compared to $10 \%$ in some studies ${ }^{(27,28)}$, and $14.6 \%-33.4 \%$ in other studies ${ }^{(18,29)}$. The variation between different studies in incidence of intraoperative iatrogenic retinal breaks is due to multifactors including the inclusion criteria, case profiles, surgeon's experience and variability between the cases even with the same diagnosis ${ }^{(27)}$.

Postoperative vitreous hemorrhage (POVCH) is one of the most common complications after PDR vitrectomy. POVCH not only prevents appropriate evaluation of the eyes in the postoperative period but also hinders the visual recovery preventing patients from resuming their daily lives ${ }^{(30)}$. POVCH can occur at any time in the postoperative period. The incidence of POVCH in PDR has significantly reduced from $75 \%$ in the 1980s to approximately 5\%-45\% as reported by Yorston et. al. due to the advances in surgical techniques ${ }^{(31)}$.

Early POVCH caused by dispersion of residual blood from the peripheral vitreous skirt and/or retinal surface as its removal without retinal and/or capsular breaks is challenging. Re-bleeding from dissected new vessels on the disc and/or on the retina may occur in the early postoperative period. Also coagula attached to the dissected new retinal vessels are often left in place to prevent re-bleeding while late $\mathrm{POVCH}$ appears to be neovascularization from residual fibro vascular tissue (32). 
Jirawison and Ittipunkul reported in their study that intravitreal injection of $1.25 \mathrm{mg}$ of bevacizumab at the end of diabetic vitrectomy decreased incidence of early postoperative vitreous hemorrhage $(\mathrm{POVCH})$ with significant visual recovery at the end of 6 months ${ }^{(33)}$.

In the current study, intraoperative bevacizumab was injected at the end of the vitrectomy operation in most cases (cases with ERM duo to diabetic retinopathy) in order to decrease early POVCH. Incidence rate of $\mathrm{POVCH}$ in the first week after PPV was $18.6 \%$, with $11.6 \%$ having minimal hemorrhage, and $7 \%$ with significant hemorrhage, of which 2 cases $(4.7 \%)$ had significant hemorrhage under SO and one case $(2.3 \%)$ had significant hemorrhage under SF6.

The choice of the proper tamponade was determined at the end of vitrectomy in each case according to severity of the case, presence of active intraoperative bleeding, retinal status and retinal breaks.

Lens-sparing vitrectomy often results in postoperative formation of nuclear sclerotic cataracts, cataract may be induced intraoperative duo to lens touch which could be avoided by orientation of the instruments. Cataract commonly progressed within one year following otherwise unremarkable vitrectomy, but younger patients and probably diabetic patients are more resistant to progressive nuclear sclerosis ${ }^{(34)}$. Holekamp et al. reported that nuclear sclerosis after lens-sparing vitrectomy for diabetic retinopathy was less progressive than in other diseases due to the presence of ischemia and low oxygen supply ${ }^{(35)}$.

In our study; out of forty three cases; LS-PPV operations were done in 29 case $(67.4 \%)$, while combined phacoemulsification PPV operations were done in 12 cases $(27.9 \%)$. There was statistical evidence of development of cataract in 18 cases of the 29 cases that had undergone LS-PPV ( $41 \%$ of the total number of cases involved in the study representing $62 \%$ of the LS-PPV group). Development of cataract had been observed after 12 to 18 months postoperatively follow up with the mean period of development of cataract was 14.6 months. Moreover, it is statistically obvious that SO has significant effect on the development/or aggravation of cataract as all cases with silicone oil had cataract after a period of follow up.

\section{REFERENCES}

1. Fraser-Bell S, Guzowski M, Rochtchina E, Wang JJ, Mitchell P(2003):Five-year cumulative incidence and progression of epiretinalmembranes: the Blue Mountains Eye Study. Ophthalmology, 110:34-40.
2. Nicholson BP, Zhou M, Rostamizadeh M et al.(2014): Epidemiology of epiretinal membrane in a large cohort of patients with uveitis.Ophthalmology, 121(12):2393-2398.

3. Kozak I, Vaidya V, Van Natta ML, Pak JW, May KP, Thorne JE(2014): The prevalence and incidence of epiretinal membranes in eyes with inactive extramacular CMV retinitis. Invest Ophthalmol Vis Sci. ,55(7):4304-4312.

4. Klein R, Klein BE, Wang QM, Moss SE(1994): The epidemiology of epiretinal membranes.Trans $\mathrm{Am}$ Ophthalmol Soc.,92:403-425.

5. Ng CH, Cheung N, Wang JJ(2011): Prevalence and risk factors for epiretinal membranes in a multi-ethnic United States population.Ophthalmology, 118(4):694-699.

6. Zhao XY, Xia S, Chen YX(2017): Antivascular endothelial growth factor agents pretreatment before vitrectomy for complicated proliferative diabetic retinopathy: a metaanalysis of randomised controlled trials. $\mathrm{Br} J$ Ophthalmol., 102:1077-1085.

7. Asato R, Yoshida S, Ogura A(2013): Comparison of gene expression profile of epiretinal membranes obtained from eyes with proliferative vitreoretinopathy to that of secondary epiretinal membranes. PLOS ONE, 8(1): 54-191.

8. Stevenson W, Prospero Ponce CM, Agarwal DR, Gelman R, Christoforidis JB(2016): Epiretinal membrane: optical coherence tomography-based diagnosis and classification.Clin Ophthalmol.,10:527-534.

9. Fraser-Bell S, Ying-Lai M, Klein R, Varma R(2004): The Los Angeles Latino Eye Study Group. Prevalence and associations of epiretinal membranes in latinos: the Los Angeles Latino Eye Study. Invest Ophthalmol Vis Sci., 45(6):1732-1736.

10. Hillenkamp J, Saikia P, Gora F (2005): Macular function and morphology after peeling of idiopathic epiretinal membrane with and without the assistance of indocyanine green. Br J Ophthalmol.,89(4):437-443.

11. Niwa T, Terasaki H, Kondo M, Piao CH, Miyake Y(2003): Function and morphology of macula before and after removal of idiopathic epiretinal membrane. Ophthalmo Vis Sci.,44(4):1652-1656.

12. Canan H, Sizmaz S, Altan-Yaycioglu R(2013): Surgical results of combined pars plana vitrectomy and phacoemulsification for vitreous hemorrhage in PDR.Clin Ophthalmol.,7:1597-1601.

13. Ohji M, Tano YA(1995): Membrane delamination wedge. Arch Ophthalmol., 113:1335-1336.

14. Eckardt C(2003): Twin lights: a new chandelier illumination for bimanual surgery. Retina,23:893-894.

15. Steinmetz RL, Grizzard WS, Hammer ME(2002): Vitrectomy for diabetic traction retinal detachment using the multiport illumination system.Ophthalmology, 109:23032307.

16. Wang ZY, Zhao PQ(2011): Four-Port Bimanual Vitrectomy in Fibrovascular Membrane Removal. Retina,31(4):798-800.

17. Shen LJ, Wang ZY, Qu J, Wang QM(2009): Bimanual technique in proliferative diabetic retinopathy using an optical fiber-free intravitreal surgery system: a case control study. Eur J Ophthalmol.,19:273-279. 
18. Lolah MMA, Shaarawy AS(2014): A comparative study of $23 \mathrm{G}$ vitrectomy and bimanual surgery in the management of epiretinal membranes in diabetic eyes. Journal of Egyptian Ophthalmological Society, 107:214-219.

19.Jang SY, Choi KS, Lee SJ(2010): Chandelier retroillumination assisted cataract extraction in eyes with vitreous hemorrhage. Arch Ophthalmol., 128:911-914.

20.Shimada H, Nakashizuka H, Hattori T, Mori R, Mizutani Y, Yuzawa M(2009):Double staining with brilliant blue $G$ and double peeling for epiretinal membranes.Ophthalmology,116(7):1370-1376.

21. Celik E, Sever O, Horozoglu F, Yanyali A(2016): Segmentation and removal of fibrovascular membranes with high-speed $23 \mathrm{G}$ transconjunctival sutureless vitrectomy, in severe proliferative diabetic retinopathy.Clin Ophthalmol.,10:903-910.

22. Fujii YG, De Juan EJ, Humayun SM, Chang ST, Pieramici JD, Barnes A(2002): Initial experience using the transconjunctival sutureless vitrectomy system for vitreoretinal surgery.Ophthalmology, 109(10):1814-1820.

23. Grigorian RA, Castellarin A, Fegan R et al.(2003): The prevalence and incidence of epiretinal membranes in eyes with inactive extramacular CMV retinitis. Invest Ophthalmol Vis Sci.,55(7):4304-4312.

24. Shen LJ, Liu L, Wang ZY, Qu J, Wang QM(2006): Vitrectomy for severe Proliferative diabetic retinopathy: a comparison of optical fiber free intravitreal surgery system with traditional vitrectomy (Article in chinase). Zhonghua Yan Ke Za Zhi.,42:600-605.

25. Durham JT, Herman IM(2011): Microvascular modifications in diabetic retinopathy. Current Diabetes Reports, 11(4):253-264.

26. Cui J, Chen H, Lu H et al.(2018): Efficacy and Safety of Intravitreal Conbercept, Ranibizumab, and Triamcinolone on 23-Gauge Vitrectomy for Patients with Proliferative Diabetic Retinopathy. Journal of Ophthalmology, 1:11.
27. Ramkissoon YD, Aslam SA, Shah SA, Wong C, Sullivan PA(2010): Risk of Iatrogenic Peripheral Retinal Breaks in 20-G Pars Plana Vitrectomy. Ophthalmology,117(9):18251830 .

28. Dogramaci M, Lee EJK, Williamson TH(2012): The incidence and the risk factors for iatrogenic retinal breaks during pars plana vitrectomy. Eye,26:718-722.

29. Farouk MM, Naito T, Sayed KM et al. (2011): Outcomes of 25-gauge vitrectomy for proliferative diabetic retinopathy. Graefes Arch Clin Exp Ophthalmol.,249:369-376.

30.Al-Kharashi A, Galbinur T, Mandelcorn ED, Muni RH, Nabavi M, Kertes PJ(2016): The adjunctive use of preoperative intravitreal bevacizumab in the setting of proliferative diabetic retinopathy. Saudi J Ophthalmol., 30(4):217-220.

31. Yorston D, Wicham L, Benson S, Bunce C, Sheard R, Charteris $\mathbf{D}(\mathbf{2 0 0 8})$ : Predictive clinical features and outcomes of vitrectomy for proliferative diabetic retinopathy. Br J Ophthalmol.,92(3):365-368.

32.Sato T, Tsuboi $K$, Nakashima H, Emi $K(2017)$ : Characteristics of cases with postoperative vitreous hemorrhage after 25-gauge vitrectomy for repair of proliferative diabetic retinopathy. Graefes Arch Clin Exp Ophthalmol., 225(4):665-671.

33. Jirawison C, Ittipunkul N(2012): Intravitreal bevacizumab at the end of diabetic vitrectomy for prevention of postoperative vitreous hemorrhage: a comparative study. $J$ Med Assoc Thai.,95(4):136-142.

34. Thompson JT(2004): The role of patient age and intraocular gas use in cataract progression after vitrectomy for macular holes and epiretinal membranes. Am J Ophthalmol.,137(2):250-257.

35. Holekamp NM, Bai F, Shui YB, Almony A, Beebe DC(2010): Ischemic diabetic retinopathy may protect against nuclear sclerotic cataract. Am J Ophthalmol.,150(4):543-550. 